\title{
Microglia exosomes miR-7239-3p promoting Glioma progression by regulating the Circadian genes
}

\section{Xuepei Li}

Sichuan University

Junwen Guan

Sichuan University West China Hospital

Zhou Jiang

Sichuan University

Shuting Cheng

Sichuan University

Wang Hou

Sichuan University

Junjie Yao

Wuhan Third Hospital

Zhengrong Wang ( $\nabla$ zhengrongwang@yeah.net)

Sichuan University https://orcid.org/0000-0002-1342-7762

\section{Research}

Keywords: Glioma, Microglia, Bmal1, Exosomes, miR-7239-3p

Posted Date: April 2nd, 2020

DOI: https://doi.org/10.21203/rs.3.rs-19744/v1

License: (c) (i) This work is licensed under a Creative Commons Attribution 4.0 International License. Read Full License 


\section{Abstract}

\section{Background}

Glioma associated microglial cells (GAMs), as an important component of the tumor microenvironment (TME) play an important role in glioma progression.

\section{Materials \& Methods}

Mouse glioma cells line GL261 and mouse microglia cell line BV2 were chosen in this studys. Firstly, Circadian genes expressions in glioma cells among M1 and M2 microglia, were detected, and unpolarized and M2 microglia exosomes were extracted. Subsequently, we labeled exosomes with PKH-67 and treated them with GL261 cells to observe the exosomes distribution. The GL261 cells phenotype and related protein expression were detected to explore the promotion effect of M2 microglial exosomes on gliomas. Then the miR-7239-3p specific inhibitors was added to vertify miR-7239-3p functions. Finally, we used a mouse subcutaneous tumorigenic model to verify the tumorigenic effect of M2 microglial exosomes in vivo.

\section{Results}

In the glioma co-cultured with M2 microglia, the expression of BMAL1 protein was decreased $(p<0.01)$ and the expression of CLOCK protein was increased $(p<0.05)$, which is in contrast to the gliomas cocultured with M1 microglial. After treated by M2 microglia exosomes, the apoptosis of GL261 cells decreased ( $p<0.001)$, and cell viability, proliferation and migration of GL261 cell increased. The expression of $\mathrm{N}$-cadherin and Vimentin increased, and E-cadherin decreased after treated by M2 microglia exosomes. When added miR-7239-3p inhibitors to M2 microglia exosomes the aforementioned results were reversed.

\section{Conclusions}

We found that miR-7239-3p in the glioma microenvironment is recruited to glioma cells by exosomes and inhibited Bmal1 expression. M2 microglial exosomes promote the proliferation and migration of gliomas by regulating tumor-related protein expression and reducing apoptosis.

\section{Background}

Glioma is the most common primary tumor in the central nervous system (CNS). The prognosis of glioma patients is closely related to the malignant degree. Currently, the prognosis of high-grade glioma patients is still not optimistic[1]. Glioma associated microglial cells (GAMs), as an important component of the tumor microenvironment (TME) in the CNS, play an important role in tumor progression [2]. According to its biological functions, GAMs are divided into M1 microglia with anti-tumor function, and M2 microglia which promoting tumor growth and invasion $[3,4]$. 
Extracellular microRNAs (miRNAs) can be transmitted stably in TME by exosomes. Exosomes miRNAs, as important communication mediators in multiple pathological environments, are involved in frequent twoway communication between tumor cells and $\operatorname{TME}[5,6]$. Recently, based on the rapid development of biotechnology, especially the high-throughput sequencing technology, the regulatory mechanism of TME have been increasingly concerned. As an important role in epigenetic regulation, miRNAs have made great progress in a variety of diseases, including tumor[7-9]. However, due to the complexity of TME, the interaction between microglia in TME and glioma, as well as the regulatory mechanism of exosomes miRNAs in specific physiological systems, still need to be further studied.

As one of the most basic physiological regulation system, circadian rhythm system widely involved in the various activities in human body and maintain the internal balance. Therefore, the disorder of the circadian rhythm system will lead to the imbalance of the internal environment and cause a variety of diseases[10-12]. Because of the complexity and universality of the circadian rhythm system, the study of its molecular mechanism is full of great challenges. Although there is increasing evidence of interaction between the immune system and the circadian rhythm system $[13,14]$, it is still unknown that, in TME, how the miRNAs and clock genes are linked together to promote glioma progression.

What we have been thinking about is that whether the glioma TME can regulate the expression of clock genes through exosomes, and affecting the proliferation and metastasis of glioma. As a core gene of the rhythm system, Bmal1 genes has been reported to be associated with the development of multiple tumors. Therefore, identifying miRNAs that can regulate Bmal1 is of great significance to elucidate the molecular mechanism of M2 microglia promoting glioma progression, and providing more theoretical basis for the biological targeted therapy of glioma.

\section{Methods And Materials}

\section{Cell culture}

The mouse glioblastoma cell line GL261 and mouse microglia cell line BV2 were purchased from the American Type Culture Collection (ATCC; Manassas, VA, USA). The GL261 and BV2 cell line was cultured in Dulbecco's modifed Eagle's medium (DMEM; Gibco, Waltham, MA, USA) supplemented with 1\% penicillin/streptomycin (Thermo Fisher Scientific, Inc.) and 10\% fetal bovine serum (FBS; Gibco, Waltham, MA, USA). Cells were cultured at $37^{\circ} \mathrm{C}$ in a humidifed incubator containing $5 \% \mathrm{CO}_{2}$.

\section{Preparation of M1/M2 phenotype TAMs and coculture}

Interferon (IFN)-y/lipopolysaccharide (LPS) and Interleukin (IL)-4 were applied to generate M1 and M2 phenotypes of BV2 cell line in vitro. LPS and IL-4 were purchased from Abcam (Cambridge, MA, USA). The BV2 cells $\left(1 \times 10^{6}\right.$ cells $\left./ \mathrm{mL}\right)$ were seeded into the upper insert of a six-well Transwell plate (Corning Inc., Corning, MA, USA) and were cultured for $6 \mathrm{~h}$ at $37^{\circ} \mathrm{C}$, followed by incubation with LPS and IL-4 
$(20 \mathrm{ng} / \mathrm{ml})$ for an addition $24 \mathrm{~h}$ at $37^{\circ} \mathrm{C}$. The samples were then washed with PBS (Phosphate Bufered Saline) and were co-cultured with GL261 cells ( $2 \times 10^{5}$ cells per well) without direct contact for $48 \mathrm{~h}$ at $37^{\circ} \mathrm{C}$. The cocultured GL261 cells were then washed and harvested for subsequent experiments.

\section{lentivirus transfection of shRNA to cells}

Bmal1 knockdown was performed by RNA interference using lentiviral transfection. Hanbio (Shanghai, China) provided the services for the synthesis of lentivirus particles that express short hairpin RNA specific for mouse Bmal1 (Bmal1 shRNA) or scrambled oligonucleotides (control shRNA). GL261 cells were seeded into 6-well plates with antibiotic-free medium overnight and transfected at 30-50\% confluence with lentivirus at an MOl of 100 . At $48 \mathrm{~h}$ after transfection, puromycin $(5 \mu \mathrm{g} / \mathrm{ml})$ was used to treat the cells for 7 days, and knockdown efficiency was assessed by Western blot analysis.

\section{Isolation, labelling and identification of exosomes}

Cell supernatant of BV2 and M2 phenotype BV2 were collected, respectively. Then, the exosomes were extracted from the supernatant according to the instructions of the ExoQuick-TC kit (Shanran Biotechnology Co., Ltd., Shanghai, China) and suspended with PBS. purified exosomes were labelled with a PKH-67 kit (Sigma, USA). Exosome specific markers CD9 were detected by Western blot analysis. The distribution of exosomes was observed using fluorescence microscope (Olympus, Japan).

\section{RNA Isolation and Quantitative Real-Time PCR}

The total RNAs of GL261 cells were purifed and enriched with a TRIzol RNA Isolation Kit (Thermo). miRNAs were extended by stem-loop methods, and total RNA reverse transcription was performed with the TransScript First-Strand cDNA Synthesis Kit (Thermo). After reverse transcription, quantitative realtime PCR was performed to analyze the levels of miRNA and mRNA transcripts using specifc primers and the QuantiTect SYBR-Green PCR kit (Qiagen, Duesseldorf, Germany). $\beta$-actin and U6 small RNA levels were used to normalize the mRNAs and miRNAs respectively. The expression levels were presented according to the $2^{-\Delta \Delta C t}$ method. Primers are listed in Supplementary Table 1.

\section{Western Blot}

Total proteins were extracted from GL261 cells with sodium dodecyl sulphte SDS polyacrylamide gel electrophoresis (SDS-PAGE) sample buffer (pH 6.8 TrisHCl, SDS, glycerol, 2-mercaptoethanol). Proteins were separated by standard SDS-PAGE using the gel system (Bio-Rad, USA) and transferred to polyvinylidene difluoride (PVDF) membranes by a transfer apparatus (Bio-Rad, USA). Membranes were incubated in blocking buffer ( $5 \%$ non-fat milk in TBST buffer) at room temperature for $2 \mathrm{~h}$ and then incubated with primary antibodies (1:2000-1:5000) overnight at $4^{\circ} \mathrm{C}$. After incubation with secondary antibodies, proteins bands were detected by enhanced chemiluminescence according to the manufacturer's instructions (Image-Quant LAS 4000 mini, USA). Image J 1.51 (National Institutes of Health, Bethesda, USA) was used for the gray analysis and the relative expressions of proteins were normalized to that of GAPDH.

\section{Cell Counting Kit8 assay}


1000 GL261 cells were seeded in a 96-well plate and incubated in complete medium. After the cells had been cultured for $0,24,48,72$ and $96 \mathrm{~h}$, or treated with exosomes, cell vitality was measured by the Cell Counting Kit-8 (CCK-8; Dojindo, Tokyo, Japan) assay on the basis of the manufacturer's instructions.

\section{Flow cytometry}

The apoptosis assay was performed with an Annexin-VFITC/PI apoptosis detection kit (Sigma-Aldrich, St. Louis, MO, USA) according to the manufacturer's manual. GL261 cells after infection or cocultured with M2 phenotype TAMs for 48 hours were washed by twice with PBS buffer. The cells were resuspended in $1 \times$ binding buffer at a concentration of $1 \times 10^{6} \mathrm{cell} / \mathrm{sL}$, and $5 \mu \mathrm{L}$ of Annexin-V-FITC conjugate and 10 $\mu \mathrm{L}$ of propidium iodide $(\mathrm{PI})$ solution were added to each $500 \mu \mathrm{L}$ cell suspension. Cells were stained by AnnexinV-FITC/PI for $30 \mathrm{~min}$ at room temperature. Stained samples were analyzed using a fowcytometer (Partec CyFlow Space, Germany), and the apoptosis rate was evaluated using Cell Quest Pro software (Qume Drive, San Jose, CA, USA).

\section{Colony forming assay}

1000 GL261 cells were plated in 6-well plates after the lentivirus transfection or treated with exosomes. After the clone was formed, the cells were washed twice with PBS, then stained with $0.1 \%$ crystal violet (Baisibio, Hangzhou, China), and the numbers of colonies per well were counted.

\section{Transwell assay}

The cell invasion ability was measured by using Transwell chambers (8- $\mu$ m pore; Coning, USA). GL261 cells $\left(1 \times 10^{5}\right.$ cells $\left./ \mathrm{mL}\right)$ were suspended of serum-free culture medium with or without exosomes and added to the upper chamber. The lower chamber was added with complete medium $600 \mu \mathrm{L}$ per well. After $48 \mathrm{~h}$, the non-invading cells on the upper surface were wiped off, and the cells that had invaded to the bottom of the membrane were fixed with methanol and stained with $0.1 \%$ crystal violet. Digital image acquisition was performed after air drying and the number of invasive cells was counted by microscope.

\section{Wound healing assay}

GL261 cell migration capacity was measured by wound healing assay. After the Culture Insert (Ibidi, Martin Reid, Germany) fixed, GL261 cells (2000 per well) were seeded in 24-well plates and cultured in complete medium with exosomes. After 48 hours, the Culture Insert was removed and the cells were washed with PBS. Cells were observed under the microscope $(0 \mathrm{~h})$, and the cells were observed again $(12 \mathrm{~h})$ after continued culture for $12 \mathrm{~h}$. Images of the plates were taken using a Nikon inverted microscope (Nikon, Tokyo, Japan) with an attached digital camera and their scratch area were quantitated with ImageJ software. The wound healing percentage was analyzed as an observational indicator. Wound healing percentage $=(0 \mathrm{~h}$ scratch area $-12 \mathrm{~h}$ scratch area $) / 0 \mathrm{~h}$ scratch area.

\section{Animal studies}

A total of 18 nude mice [8-week-old male, weighing $(22 \pm 2)$ g] were provided by the Sichuan University Laboratory Animal Center (Sichuan, China). The mice were fed in a humidified condition $(55 \pm 10 \%)$ at the 
temperature of $(21 \pm 2) \mathrm{C}$ with a cycle of $12 \mathrm{~h}$ light/dark. The cell line GL261 in log phase of mouse glioma was implanted subcutaneously in nude mice. After 7 days, the three groups of mice were intravenously injected with PBS or equal volumes of BV2 exosomes or M2 phenotype BV2 through the tail vein.

Subsequently, The body weight of nude mice was recorded every 3 days after cell injection, as well as the tumor length and diameter. The tumor volume was calculated as (length $\times$ diameter $\left.^{2}\right) / 2$. After fixed with $4 \%$ paraformaldehyde (PFA), tumor tissues were processed for paraffin embedded blocks.

\section{Histopathological and Immunohistochemistry analyses}

At the end of the study period, the tumor tissues embedded in paraffin were cut into five micrometer-thick sections and stained with hematoxylin and eosin (H\&E) to detect histomorphometry observation. In the immunohistochemistry (IHC) assay, the tumor tissues were stained with debranching enzyme (DBE)conjugated anti-Bmal1 (Abcam, USA). The fluorescence intensity was quantified from at least five sections. All samples were observed using an inverted microscope (Nikon Eclipse Ti, Nikon, Tokyo, Japan).

\section{TUNEL assay}

To investigate the tumor tissue apoptosis, the terminal deoxynucleotidyl transferase (TdT)-mediated dUTP nick end-labeling (TUNEL) assay was performed by the cell death detection kit (Roche, San Francisco, USA). In detail, the tumor tissue sections were deparaffinized and rehydrated, and then maintained in the TUNEL reaction mixture for $60 \mathrm{~min}$ and Converter-POD solution for $30 \mathrm{~min}$ in dark at 37C. Subsequently, the samples were exposed to diaminobenzidine (DAB) substrate for 6 min and observed under the Leica photograph microscope. Apoptosis index was used to evaluate the apoptosis of mice in each group. The apoptosis index $=$ (number of apoptotic cells/total number of cells) $\times 100 \%$.

\section{Statistical analysis}

All of the statistical analyses were carried out by using GraphPad Prism 6. Values were presented as the mean \pm SD with of least three independent experiments in triplicate. Student's $t$ test was used for pantwise comparison of data. one-way ANOVA was used for comparison of mean between multiple groups, and Pearson test was used for correlation analysis of mRNAs. The statistical significance parameter was set at $\mathrm{P}<0.05$.

\section{Results}

\section{M1/M2 phenotype microglia regulate circadian gene expression of glioma}

LPS and IL-4 were used to induce BV2 to M1/M2 phenotype, respectively. After $24 \mathrm{~h}$, the expression of microglial markers was detected by Real-time QPCR. The results showed that the expression of M1 phenotype markers, IL-12 $(p<0.05)$ and iNOS $(p<0.01)$ were increased after LPS treatment. And M2 
phenotype markers, Arg-1 and IL-4 was increased as well ( $p<0.01)$ (Fig. 1A). These indicate the M1/M2 phenotype were successfully induced.

M1/M2 phenotype BV2 were co-cultured with glioma cells GL261, and the protein expression of circadian genes Clock and Bmal1 in GL261 cells was detected by Western blot 48 hours later. The results showed that the expression of BMAL1 protein in the BV2(M1) + GL261 group was increased $(p<0.01)$, and in the $B V 2(M 2)+G L 261$ group was significantly reduced $(p<0.01)$. On the contrary, the expression of CLOCK protein in the BV2(M1) + GL261 group was reduced ( $<<0.05)$, and in the BV2(M2) + GL261 group was increased $(p<0.05)$ (Fig. 1B,C).

\section{M2 phenotype microglia promotes glioma proliferation}

M2 phenotype BV2 were co-cultured with glioma cells GL261. The cell cycle of glioma was measured by PI staining after $48 \mathrm{~h}$. The results showed that the cell amount in G1 phase of the GL261 + BV2(M2) group was lower than that of the GL261 + BV2 group $(p<0.01)$. While the cell amount in $G 2 / M$ and $S$ phases was higher than that of the GL261 + BV2 group $(p<0.05, p<0.001)$ (Fig. 2A,B). The survival rate of glioma cells was continuously measured for 5 days. The results showed that the cell survival rate in the GL261 + BV2(M2) group was higher than that in the GL261 + BV2 group ( $<<0.01, p<0.001)$ (Fig. 2C). These results suggest that M2 phenotype microglia improves the survival rate of glioma cells and promote its proliferation.

\section{Down-regulation of Bmal1 promotes proliferation and migration of GL261 cells}

The infection effect of lentiviral interfering sequences was observed by the expression level of BMAL1 protein. The results showed that BMAL1 protein expression was significantly reduced in the sh-Bmal1 group, and there was no difference in BMAL1 protein expression in the Blank group and the sh-NC group $(p<0.05)(F i g .3 A, B)$. The results suggest that we have successfully integrated the interference sequence of Bmal1 into the genome of GL261 cells.

The results of the transwell assay showed a significant increase in the number of cells crossing the diaphragm in the sh-Bmal1 group $(p<0.05)(F i g .3 C, E)$. Colony forming assay results showed that the shBmal1 group displayed much larger and more colonies $(p<0.05)$ (Fig. 3D,F). Therefore, down-regulation of Bmal1 gene promotes the proliferation and migration ability of GL261 cells in vitro.

\section{Up-regulated miR-7239-3p in M2 phenotype microglia exosomes}

Firstly, CD9 was detected to verify the extracted microglia exosomes. The results showed that CD9 protein was significantly expressed in both groups of exosomes (Fig. 4A,B), indicating that microglia exosomes were successfully isolated. Targetscan are used to predict miRNAs that target Bmal1 3'UTR, and miR7239-3p was selected. RT-qPCR was used to detect miR-7239-3p expression in exosomes. The results 
showed that the expression of exosomes miR-7239-3p in M2 microglia was significantly up-regulated. (Fig. 4C).

In order to explore how microglial-derived exosomes interact with gliomas, we labeled exosomes with PKH-67 and observed its distribution in gliomas. The results showed that exosomes were evenly distributed in the cytoplasm of glioma cells after incubation for 12 hours (Fig. 4D). These evidences suggest that glioma cells are able to take up microglial exosomes, and are likely to be achieved by the cell's endocytosis. Moreover, the up-regulated miR-7239-3p expression in glioma cells is closely related to the M2 phenotype microglia exosomes.

\section{M2 phenotype microglia exosomes promote glioma progression}

After glioma cells were incubated with microglia exosomes and M2 phenotype microglia exosomes, the apoptosis and cell cycle of GL261 cells were detected by flow cytometry. The results showed that early apoptosis, late apoptosis, and total apoptosis in the GL261 + BV2(M2) Exosomes group were reduced ( $p$ $<0.01, p<0.001)$ (Fig. $5 A, B)$, the cells amount in the $G 1$ phase was reduced $(p<0.01)$, and the cells amount in the $S$ phase was increased $(p<0.01)($ Fig. 5C,D).

Subsequently, Colony forming assay, CCK8 assay was used to detect the cell proliferation ability, transwell assay and wound healing assay were used to evaluate the invasion and migration ability, respectively. The results showed that GL261 + BV2(M2) Exosomes group displayed larger and more colonies $(p<0.01)(F i g .5 E, H)$, and showed a significant increase in the number of cells crossing the diaphragm $(p<0.01)$ (Fig. 5F,I). The cell survival rate in the GL261 + BV2(M2) Exosomes group was significantly higher $(p<0.001)$ (Fig. $5 G)$. And the wound healing percentage of the GL261 + BV2(M2) Exosomes group was significantly higher than that of the GL261 + BV2 Exosomes group $(p<0.05)$ (Fig. 5J,L).

RT-qPCR was used to detect the RNAs expression of Bmal1 and miR-7239-3p in vitro, and western blot was used to detect the expression of tumor-related proteins. The RT-qPCR results showed that the expression of Bmal1 in GL261 + BV2(M2) Exosomes group was reduced ( $<<0.001)$, while the expression of miR-7239-3p was increased ( $p<0.001$ ) (Fig. 5K). Western blot results showed that the expression of BMAL1 and E-Cadherin were reduced in the GL261 + BV2(M2)Exosomes group $(p<0.05, p<0.01)$, while the expression of $\mathrm{N}$-Cadherin and Vimentin increased $(\mathrm{p}<0.05, \mathrm{p}<0.01)$ (Fig. 5M,N).

\section{miR-7239-3p Inhibitor reverses tumor-promoting effects of M2 phenotype microglia exosomes}

In order to identify that miR-7239-3p is an important intermediate molecule involved in the process of M2 phenotype microglia exosomes promoting glioma progression. We added miR-7239-3p inhibitor and inhibitor NC to M2 phenotype microglia exosomes, respectively, to observe the growth of glioma cells and the expression of tumor-related proteins. The results of flow cytometry showed that the cell amount of 
early apoptosis and total apoptosis in miR-7239-3p Inhibitor group were increased $(p<0.001)(F i g .6 A, B)$, and the number of cells in G1 and G2/M phases of this group increased $(p<0.05, p<0.01)$, the number of cells in S phase was significantly reduced $(p<0.001)$ (Fig. 6C,D). The results suggest that miR-7239-3p accelerates glioma proliferation and reduce glioma apoptosis. Furthermore, miR-7239-3p mainly affects the early apoptosis of cells, but has little effect on late apoptosis.

The results of colony forming assay showed that the miR-7239-3p Inhibitor group displayed smaller and fewer colonies $(p<0.05)(F i g .6 E, H)$, indicating a weakened cell proliferation. The results of transwell assay showed a significant decrease in the number of cells crossing the diaphragm of miR-7239-3p Inhibitor group $(p<0.01)$ (Fig. 6F,I), indicating a reduced invasion ability. The results of CCK-8 assay showed that the cell survival rate of the miR-7239-3p Inhibitor group was also significantly lower than that of the control group $(p<0.001$ ) (Fig. $6 G)$. And the wound healing percentage of miR-7239-3p Inhibitor group was significantly lower than the control group $(p<0.05)($ Fig. $6 \mathrm{~J}, \mathrm{~L})$.

The RT-qPCR results showed that the expression of Bmal1 in the miR-7239-3p Inhibitor group increased $(p<0.001)$ and the expression of miR-7239-3p decreased ( $p<0.001)$ (Fig. 6K), indicating that miR-7239$3 p$ was successfully inhibited. Western blot results showed that BMAL1 and E-Cadherin expression increased in the miR-7239-3p Inhibitor group $(p<0.01, p<0.001)$, and $\mathrm{N}$-Cadherin and Vimentin expression decreased $(\mathrm{p}<0.01)($ Fig. $6 \mathrm{M}, \mathrm{N})$.

\section{Negative correlation between Bmal1 and miR-7239-3p expression in vivo}

After killing the mice, the relative expression of Bmal1 and miR-7239-3p in tumor tissues was detected. The results showed that the expression of Bmal1 in tumor tissue of GL261 + BV2(M2) Exosomes group was reduced $(p<0.001)$, and the expression of miR-7239-3p was increased $(p<0.001)$. And in the GL261 group, Bmal1 expression was increased $(p<0.001)$, miR-7239-3p expression was decreased $(p<0.001)$ (Fig. 7A). In addition, the correlation analysis showed that miR-7239-3p was negatively correlated with Bmal1 expression $(p<0.001)$ (Fig. 7B)

\section{M2 phenotype microglia exosomes promote glioma growth in vivo}

We detected BMAL1 protein expression in tumor tissues by IHC. The results showed that the BMAL1 staining area and staining intensity in tumor tissues were reduced in GL261 + BV2(M2) Exosomes group. By statistical analysis, the expression of BMAL1 protein in this group was significantly reduced $(p<0.05$, $p<0.001)$ (Fig. 8A,B). This result is in accordance with the RNA results. These evidence further suggested that M2 phenotype microglia exosomes are likely to affect the expression of miR-7239-3p in vivo, and further effects on downstream regulators by down-regulating the expression of BMAL1 protein.

On the 30th day after glioma injection, we killed the mice and took pictures and recorded the tumor volume (Fig. 8I). The results showed that in the GL261 + BV2(M2) Exosomes group, the tumors wight and 
volume increased faster $(p<0.05, p<0.01)(F i g .8 F, H)$. And the mice weight in the GL261 + BV2(M2) Exosomes group had no significant increase throughout the study, while the other two groups mice displayed a significant increase from the 24th day $(p<0.01)$ (Fig. 8G).

We used HE staining to observe the morphological characteristics of tumor cells in each group, and observed apoptosis in glioma tissue by TUNEL assay. The results showed that in the GL261 + BV2(M2) Exosomes group, the microglioma cells were closely arranged with normal size and the clear structure. Both the GL261 group and the GL261 + BV2 Exosomes group displayed reduced cell bodies and large gaps, and some cell nuclear showed shrinking and with fragmentation(Fig. 8C). TUNEL assay results showed that a large number of apoptosis was seen in the GL261 group and GL261 + BV2 Exosomes group, and the apoptosis index were $(42.93 \pm 10.07) \%$ and $(48.90 \pm 7.98) \%$, respectively. The GL261 + BV2 (M2) Exosomes group showed a significantly reduced apoptosis bodies $(p<0.001)$, and the apoptosis index was $(19.43 \pm 5.09) \%$ (Fig.D,E).

\section{Discussion}

GAMs are the largest part of tumor infiltrating cells, accounting for $30-70 \%$ of the volume of gliomas. The cytokines and chemokines produced by GAMs help tumor growth and maintain the immunosuppressive microenvironment[15]. Lisi et al. studied the activation of microglia in clinical samples from 41 patients with grade IV glioblastoma. By analyzing the tumor center and surrounding parenchyma of each patient, four markers of IBA-1, CD163, iNOS, and ARG-I were tested, and M2 microglial markers were considered as prognostic markers, instead of M1[16]. This indicates that M2 microglial cells are an important part of glioma-mediated immune evasion. Therefore, we chose M2 microglia for further research. We found that microglial cells with different polarization wound affect the expression of circadian genes in gliomas: after co-culture with M2 microglial, the expression of BMAL1 protein decreases and the CLOCK expression increases. We confirmed that the $\mathrm{M} 2$ microglial cells can promote the proliferation of glioma by the CCK-8 assay and FCM, which is consistent with the recently findings of Lisi et al[16].

In previous work, we have found that the growth of a variety of tumor cells is related to disturbances in circadian rhythms or changes in circadian genes $[17,18]$. Bmal1 gene is down-regulated in a variety of tumors and inhibits tumor growth both in vivo and in vitro [19]. Clock gene expression varies with tumor type. For example, Clock genes are under-expressed in a variety of malignant tumors such as ovarian, prostate, and pancreatic ductal carcinomas $[20,21]$. The expression of Clock in colorectal cancer and breast cancer is elevated [22]. Therefore, in our study, it seems understandable that the expression of BMAL1 protein and CLOCK protein showed the opposite trend. Finally, we used the lentiviral transfection technique to obtain a stable down-regulation of Bmal1 in GL261 cell line, and found that down-regulation of Bmal1 gene enhanced the proliferation and migration ability of glioma cells and promoted the progress of glioma.

miRNAs have great potential in developing biomarkers for tumor diagnosis and prognosis [23]. Exosomes could protect miRNAs from degradation, stably express them in the outer space of cells, and deliver them 
to specific recipient cells [24, 25]. We predicted miR-7239-3p by predicting miRNAs that might target Bmal1 3'UTR. After treating glioma cells with M2 and unpolarized microglia exosomes, we found that the expression of Bmal1 and miR-7239-3p was significantly different in the two groups. M2 microglial exosomes-treated glioma cells enhanced its growth, migration and invasion capabilities. The changes in the expression of E-Cadherin and $\mathrm{N}$-Cadherin proteins are consist with the trend in the malignant progression of most tumor cells. E-Cadherin and $\mathrm{N}$-Cadherin proteins are important cadherins during epithelial-mesenchymal transition (EMT), and EMT is the initial cause of tumor invasion and metastasis [26]. One of the marker of EMT is the loss of epithelial cells integrity, accompanied by weakened adhesion between epithelial cells. In this process, gene transcription of epithelial-specific proteins (such as ECadherin) is suppressed by EMT-promoting transcription factors, which promotes the degradation of adhesion junctions. At the same time, epithelial cell-specific proteins will be replaced by more flexible cadherin (such as $\mathrm{N}$-Cadherin), which promotes cell isolation and enhances cell mobility [27]. Our results demonstrate that Exosomes of M2 microglial down-regulate E-Cadherin and up-regulate $\mathrm{N}$-Cadherin expression, promoting the malignant process of gliomas. In addition, Vimentin negatively regulates $\mathrm{E}$ Cadherin and participate in the process of cell invasion [28], it also provides a more sufficient argument for the above results.

After adding a specific inhibitor of miR-7239-3p, the tumor-promoting effect of M2 microglia exosomes was significantly reversed. Finally, by establishing a glioma model in nude mice, we found that miR-7239$3 p$ and Bmal1 gene expression were negatively correlated. Therefore, we speculated that exosomes miR7239-3p is an important intermediate molecule in M2 microglial promoting glioma process. M2 microglial carry miR-7239-3p through exosomes, entering glioma cells by endocytosis, inhibiting Bmal1 gene expression, and ultimately promoting glioma progression.

\section{Conclusions}

In summary, we found that miR-7239-3p in the glioma microenvironment is recruited to glioma cells by exosomes and inhibited Bmal1 expression. M2 microglial exosomes promote the proliferation and migration of gliomas by regulating tumor-related protein expression and reducing apoptosis. We discovered the mechanism by which M2 microglial promote the glioma progression, and the role of M2 microglial cells exosomes miR-7239-3p in the tumor microenvironment, which may provide theoretical basis of biological treatment for glioma patients.

\section{Declarations}

\section{Ethics approval and consent to participate}

Not applicable.

\section{Consent for publication}

All authors agree to submit the article for publication. 
Availability of data and material

Not applicable.

\section{Competing interests}

No potential conflicts of interest were reported by the authors.

\section{Funding}

This study was funded by the National Nature Science Foundation of China (31371180).

\section{Author Contributions}

Xuepei Li, study concept and design, animal experiments, analysis and interpretation of data, Junwen Guan, design and interpretation data

Zhou Jiang, animal experiments

Shuting Cheng, animal experiments

Wang Hou, statistical analysis

Junjie Yao, statistical analysis

Zhengrong Wang, study supervision

\section{Acknowledgements}

We would like to express our gratitude to Dr. Qi xin for her valuable comments in this study.

\section{References}

[1] Ostrom Q T, Gittleman H, Kruchko C, Louis D N, Brat D J, Gilbert M R, et al. Completeness of required site-specific factors for brain and CNS tumors in the surveillance, epidemiology and end results (SEER) 18 database (2004-2012, varying). J Neuro-Oncol. 2016; 130(1): 31-42.

[2] Hambardzumyan D, Gutmann D H and Kettenmann $\mathrm{H}$. The role of microglia and macrophages in glioma maintenance and progression. Nat. Neurosci. 2016; 19(1): 20-7.

[3] Mantovani A, Sica A, Sozzani S, Allavena P, Vecchi A and Locati M. The chemokine system in diverse forms of macrophage activation and polarization. Trends Immunol. 2004; 25(12): 677-86.

[4] Verreck F A, de Boer T, Langenberg D M, Hoeve M A, Kramer M, Vaisberg E, et al. Human IL-23producing type 1 macrophages promote but IL-10-producing type 2 macrophages subvert immunity to 
(myco) bacteria. Proc Natl Acad Sci USA. 2004; 101(13): 4560-65.

[5] Hu G, Drescher K M, and Chen X M. Exosomal miRNAs: Biological Properties and Therapeutic Potential. Front Genet. 2012; 3: 56.

[6] Purushothaman A, Bandari S K, Liu J, Mobley J A, Brown E E and Sanderson R D. Fibronectin on the Surface of Myeloma Cell-derived Exosomes Mediates Exosome-Cell Interactions. J Biol Chem. 2016; 291(4): 1652-63.

[7] Gurunathan S, Kang M H, Jeyaraj M, Qasim M and Kim J H. Review of the Isolation, Characterization, Biological Function, and Multifarious Therapeutic Approaches of Exosomes. Cells. 2019;8(4): E307.

[8] Gabrusiewicz K, Li X, Wei J, Hashimoto Y, Marisetty A L, Ott M, et al. Glioblastoma stem cell-derived exosomes induce M2 macrophages and PD-L1 expression on human monocytes. Oncoimmunology. 2018; 7(4): e1412909.

[9] Palumbo S, Miracco C, Pirtoli L and Comincini S. Emerging Roles of microRNA in Modulating CellDeath Processes in Malignant Glioma. J Cell Physiol. 2013; 229(3): 277-286.

[10] Masri S, and Sassone-Corsi P, The emerging link between cancer, metabolism, and circadian rhythms. Nat Med. 2018; 24(12): 1795-803.

[11] Papantoniou K, Castaño-Vinyals G, Espinosa A, Aragonés N, Pérez-Gómez B and Burgos J. Night shift work, chronotype and prostate cancer risk in the MCCSpain case-control study. Int J Cancer. 2015; 137(5): 1147-57.

[12] Straif K, Baan R, Grosse Y, Secretan B, El Ghissassi F, Bouvard V, et al. Carcinogenicity of shift-work, painting, and fire-fighting. Lancet Oncol. 2007; 8(12): 1065-6.

[13] Yuan Y, Wu S, Li W, He W. A Tissue-Specific Rhythmic Recruitment Pattern of Leukocyte Subsets. Front Immunol. 2020;11.

[14] Hergenhan S, Holtkamp S, and Scheiermann C. Molecular Interactions Between Components of the Circadian Clock and the Immune System. J Mol Biol. 2020 Jan 10.

[15] da Fonseca A C and Badie B, Microglia and macrophages in malignant gliomas: recent discoveries and implications for promising therapies. Clin. Dev. Immunol. 2013: 264124.

[16] Lisi L, Ciotti G M, Braun D, Kalinin S, Currò D, Dello Russo C, et al. Expression of iNOS, CD163 and ARG-1 taken as M1 and M2 markers of microglial polarization in human glioblastoma and the surrounding normal parenchyma. Neurosci Lett. 2017; 645:106-12.

[17] Hua H, Wang Y, Wan C, Liu Y, Zhu B, Yang C, et al. Circadian gene mPer2 overexpression induces cancer cell apoptosis. Cancer Sci. 2006; 97(7): 589-96. 
[18] Li X, Wang S, Yang S, Ying J, Yu H, Yang C, Liu Y, et al. Circadian locomotor output cycles kaput affects the proliferation and migration of breast cancer cells by regulating the expression of E-cadherin via IQ motif containing GTPase activating protein 1. Oncol Lett. 2018; 15(5): 7097-103.

[19] Mazzoccoli G, Panza A, Valvano M R, Palumbo O, Carella M, Pazienza V, et al. Clock gene expression levels and relationship with clinical and pathological features in colorectal cancer patients. Chronobiol Int. 2011; 28(10): 841-51.

[20] Tokunaga H, Takebayashi Y, Utsunomiya H, Akahira J, Higashimoto M, Mashiko M, et al. Clinicopathological significance of circadian rhythm-related gene expression levels in patients with epithelial ovarian cancer. Acta Obstet Gynecol Scand. 2008;87(10): 1060-70》

[21] Relles D, Sendecki J, Chipitsyna G, Hyslop T, Yeo CJ and Arafat H A. Circadian gene expression and clinicopathologic correlates in pancreatic cancer. J Gastrointest Surg. 2013; 17(3): 443-50囚

[22] Karantanos T, Theodoropoulos G, Gazouli M, Vaiopoulou A, Karantanou C and Lymberi M. Expression of clock genes in patients with colorectal cancer. Int J Biol Markers. 2013; 28(3): 280-5.

[23] Lu J, Getz G, Miska EA, Alvarez-Saavedra E, Lamb J, Peck D, et al. MicroRNA expression profifiles classify human cancers. Nature. 2005; 435: 834-8.

[24] Cordonnier M, Chanteloup G, Isambert N, Seigneuric R, Fumoleau P, Garrido C, et al. Exosomes in cancer theranostic: Diamonds in the rough. Cell Adh Migr. 2017; 11(2): 151-63.

[25] Tkach $M$ and Théry $C$. Communication by extracellular vesicles: where we are and where we need to go. Cell. 2016; 164(6): 1226-32.

[26] Wheelock M J and Johnson K R. Cadherins as Modulators of Cellular Phenotype. Annual Review of Cell and Developmental Biology. Cell Res. 2003;19(1): 207-35.

[27] Lamouille S, Xu J and Derynck R. Molecular mechanisms of epithelial-mesenchymal transition. Nat Rev Mol Cell Biol. 2014; 15(3): 178-96.

[28] Singh S, Sadacharan S, Su S, Belldegrun A, Persad S and Singh G. Overexpression of vimentin: role in the invasive phenotype in an androgen-independent model of prostate cancer. Cancer Res. 2003; 63(9): 2306-11.

\section{Figures}



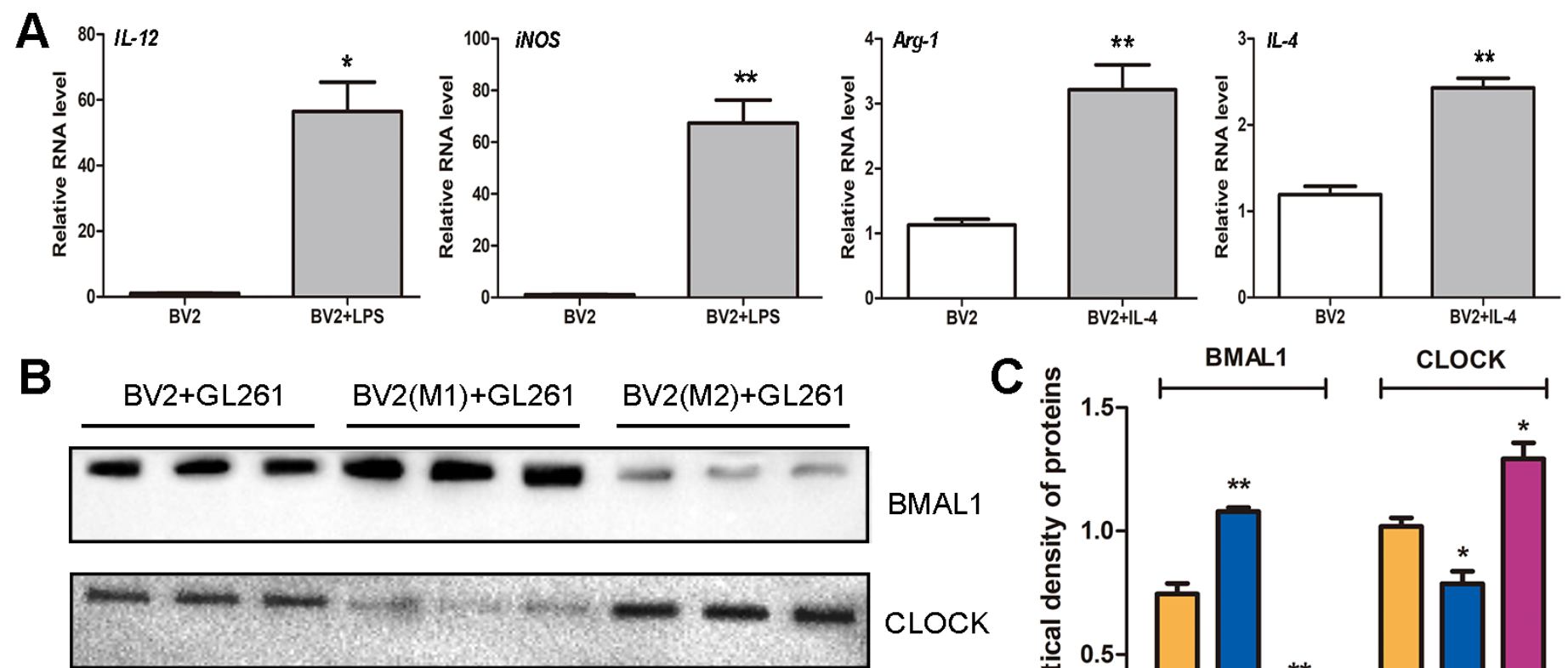

BMAL1

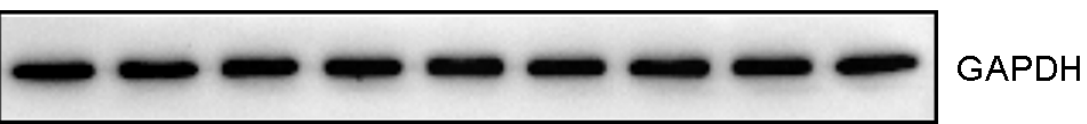

CLOCK

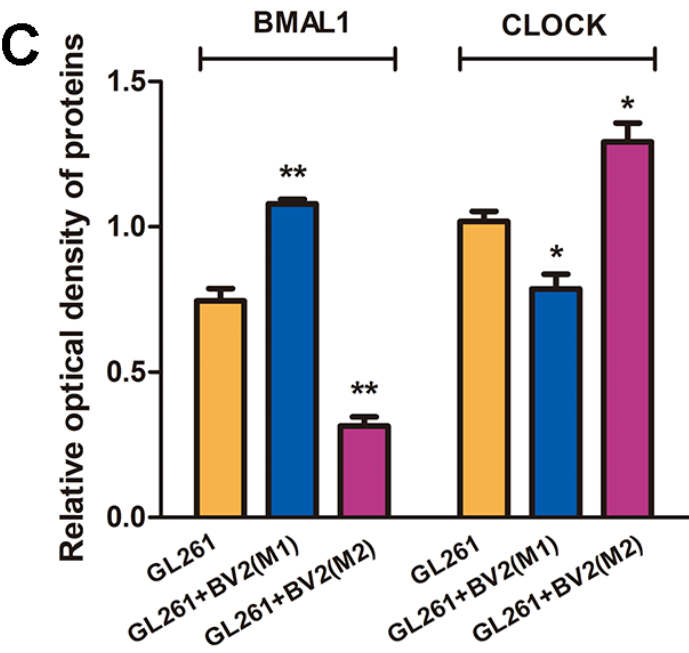

\section{Figure 1}

M1/M2 phenotype microglia regulate circadian gene expression of glioma. (A)Real-time QPCR was used to verify the expression of microglia markers in BV2 cells. The internal reference gene was $\beta$-actin.

(B)Wstern Blot was used to verify the expression of two core clock genes in three groups of GL261 cells.

(C)The results of optical density analysis. The internal reference protein is GAPDH. ${ }^{\star} p<0.05,{ }^{\star \star} p<0.01$. 

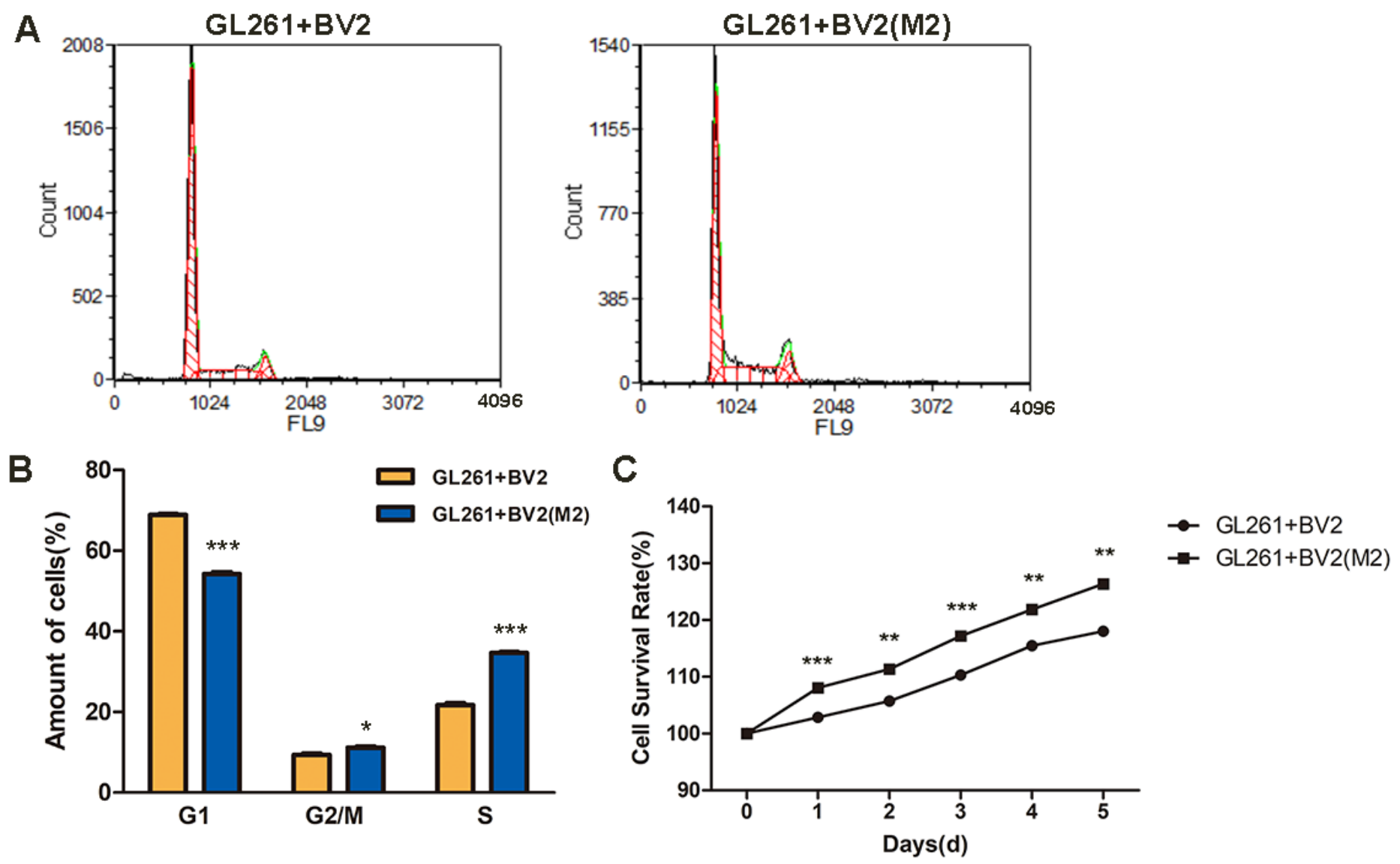

\section{Figure 2}

M2 phenotype microglia promotes glioma proliferation. (A)Cell cycle of GL261 was detected by flow cytometry(FCM) following PI staining. The abscissa represents the DNA content and the ordinate represents the cell counts. (B)The results of cell cycle analysis. The white bar represents the GL261+BV2 group and the gray represents the GL261+BV2(M2) group. (C)Survival curve of GL261 cells within 1-5 days after co-culture (CCK-8 assay). The abscissa is the number of days and the ordinate is the cell survival rate. ${ }^{\star} p<0.05,{ }^{* \star} p<0.01,{ }^{* \star *} p<0.001$. 


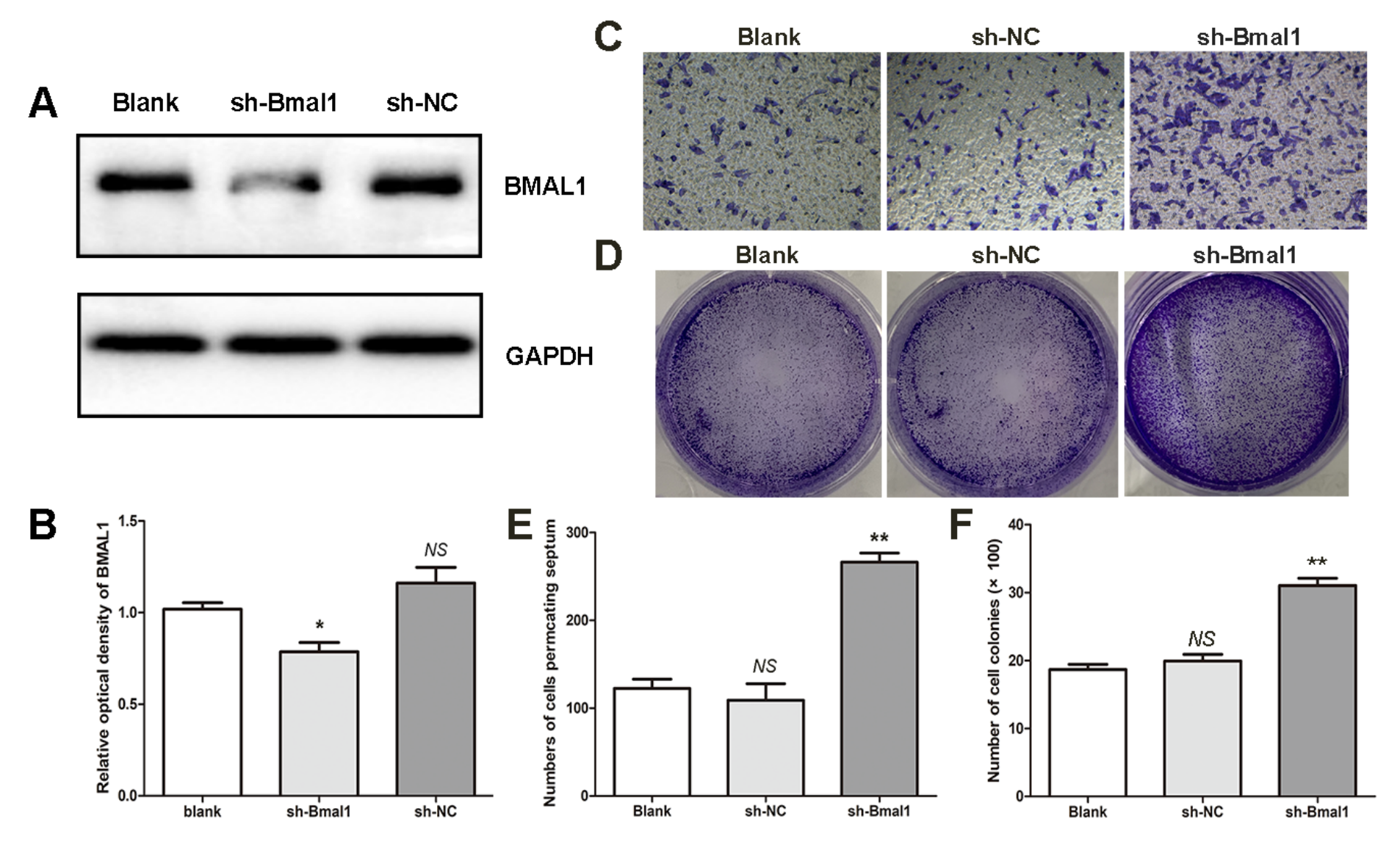

Figure 3

Establishment of down-regulated Bmal1 in GL261 cell and its effect on glioma proliferation. (A)Western blot was used to detect the changes in the expression level of BMAL1 protein in the three groups of cells. (B)The result of optical density analysis. The internal reference protein is GAPDH. Blank was the Blank control group, sh-NC was the negative control group transfected with HBLV-GFP-PURO, sh-Bmal1 was the study group transfected with the Bmal1 interfering virus HBLV-Arntl-shRNA-GFP-PURO. Transwell Assay was used to measure the cell migration.(C) is the microscopic image of GL261 cells penetrating the chamber after crystal violet staining $(\times 100)$. The results that plate clone formation assay was used to measure the cell proliferation. (D) shows the formed clones of GL261 cells after crystal violet staining. (E)The statistical result of the numbers of cells penetrating the chamber in each group. (F)The statistical results of the clones formed number in each group. ${ }^{*} \mathrm{p} \otimes 0.05,{ }^{*} \mathrm{p}<0.01$. 

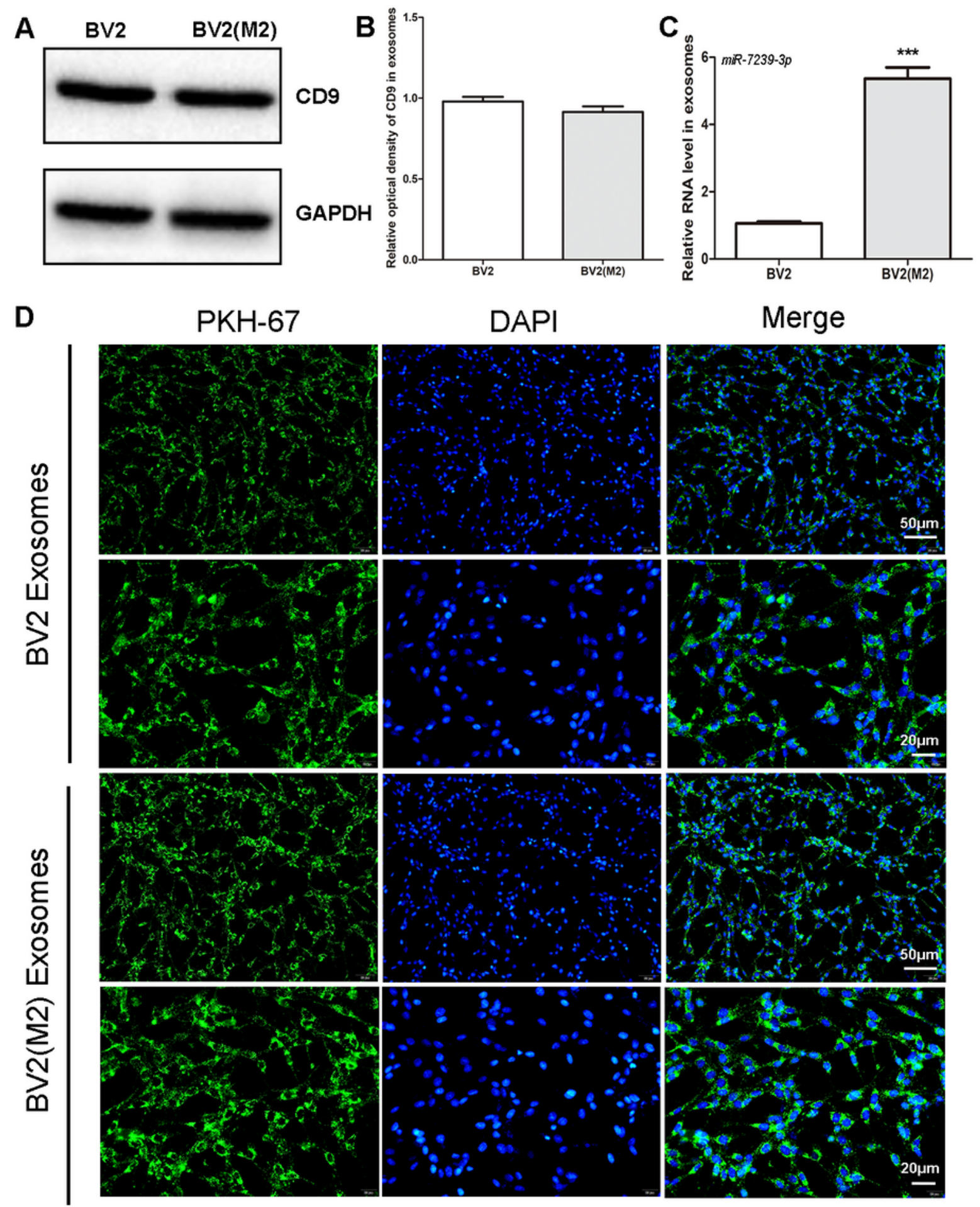

\section{Figure 4}

Distribution and identification of exosomes and expression of miR-7239-3p. (A)Wstern Blot was used to verify the expression of marker CD9 in the exosomes of two groups. (B)The results of optical density analysis. The internal reference protein is GAPDH. (C)Real-time QPCR verified the expression of exosome miR-7239-3p in two groups of microglia cells. The internal reference gene was U6. ${ }^{* \star} p \llbracket 0.001$. (D)The 
localization of exosomes in GL261 cells was observed by fluorescence microscope. DAPI for nuclear staining, blue fluorescence; $\mathrm{PKH}-67$ for exosomes labeling, green fluorescence.

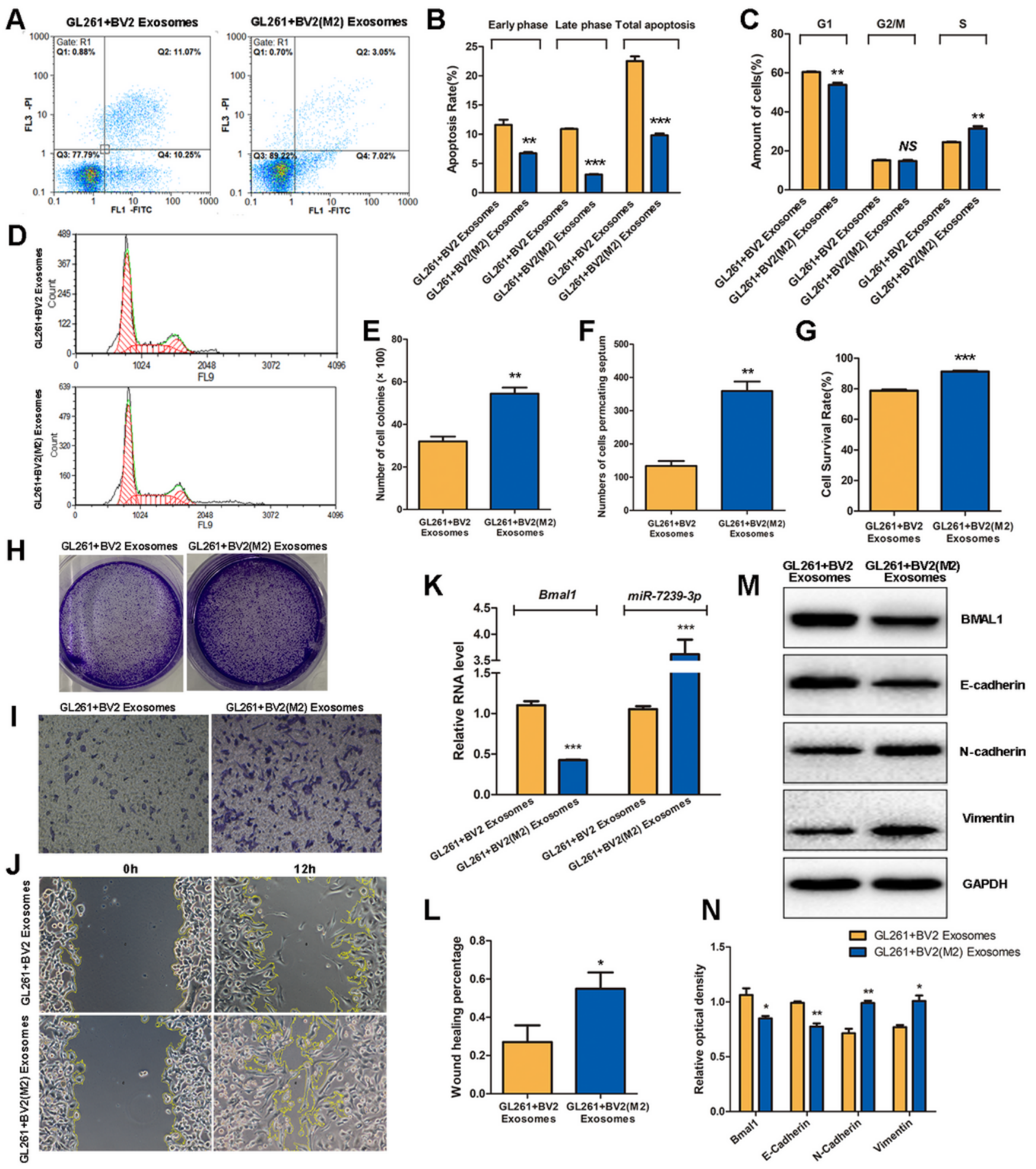

Figure 5

M2 phenotype microglia exosomes promote glioma progression. Cell apoptosis and cell cycle of GL261 was detected by FCM following PI staining. $(A, B)$ is FCM results of Apoptosis. The abscissa represents Annexin V, and the ordinate represents PI. (C,D) is FCM results of cell cycle. The abscissa represents the 
DNA content and the ordinate represents the number of effective cells. (E)The statistical results of the clones formed number in each group. (F)The statistical result of the numbers of cells penetrating the chamber in each group. (G)The statistical results of CCK8 assay for cell survival. (H)The formed clones of GL261 cells after crystal violet staining. (I)The microscopic image of GL261 cells penetrating the chamber after crystal violet staining (×100). (J)The microscopic image of GL261 cell scratch experiments. (K)The relative expression levels of Bmal1 and miR-7239-3p RNA in the two groups were detected by real-time QPCR. The internal reference protein is $\beta$-actin. $(\mathrm{L})$ The statistical results of cell scratch experiments. The vertical axis represents the wound healing percentage. (M)Protein bands of relevant proteins in the two groups were detected by Western blot. (N)Quantitative analysis of protein expressions. ${ }^{\star} \mathrm{p} \otimes 0.05,{ }^{\star *} \mathrm{p} \otimes 0.01,{ }^{\star * \star} \mathrm{p} \otimes 0.001$. 
A

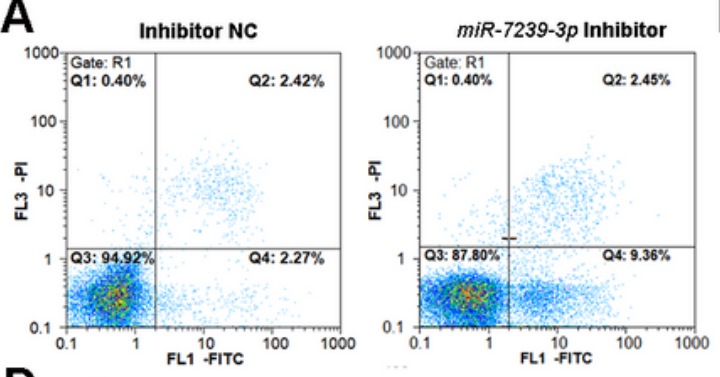

D
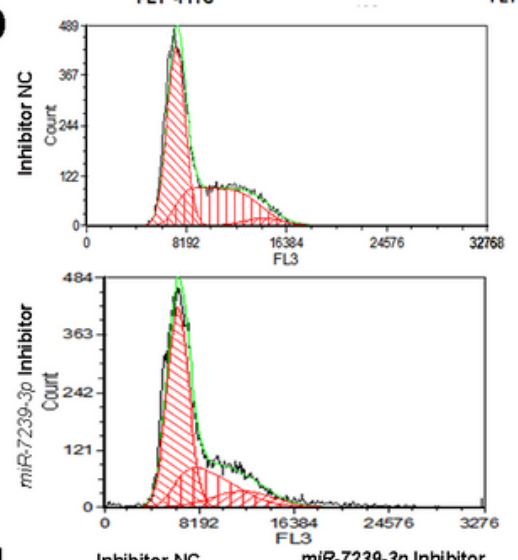

H

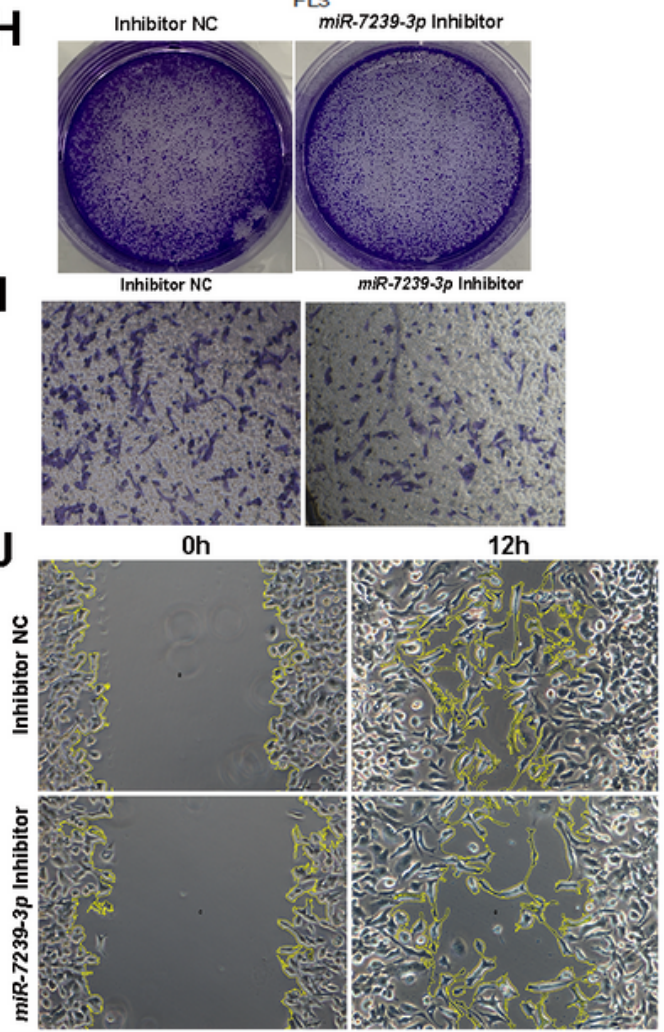

B Early phase Late phase Total apoptosis

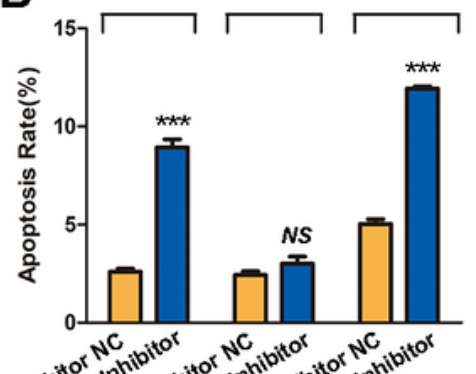

${ }^{C} \mathrm{C}$
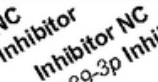

min $x^{-12^{2}}$

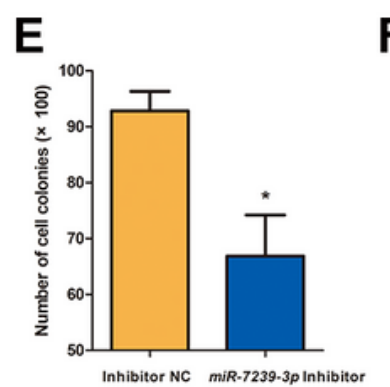

$F$

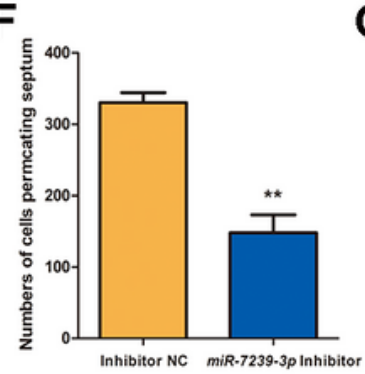

G
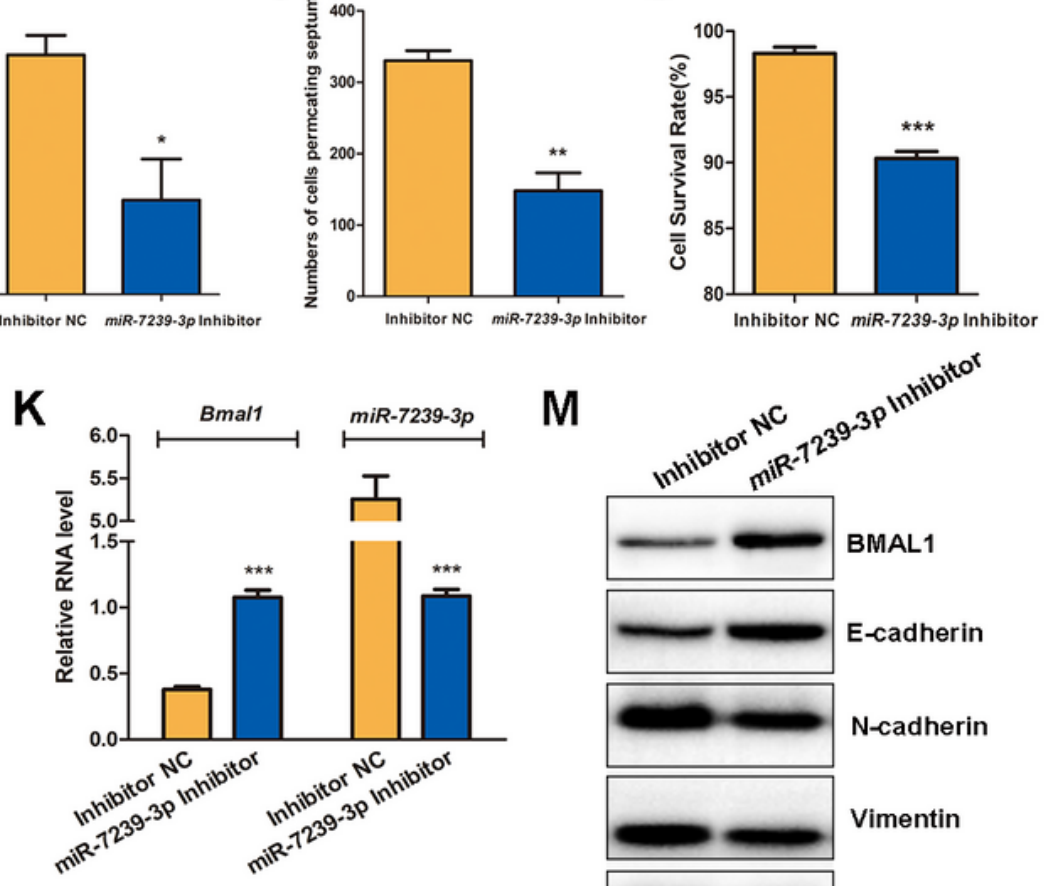

M
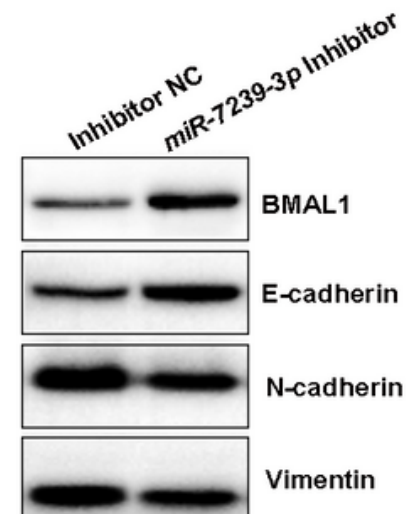

L

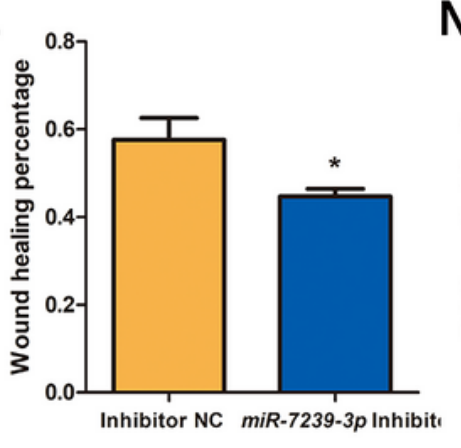

N

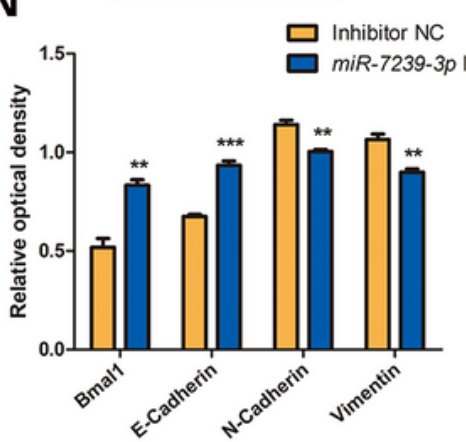

\section{Figure 6}

miR-7239-3p Inhibitor reverses tumor-promoting effects of M2 phenotype microglia exosomes. Cell apoptosis and cell cycle of GL261 was detected by FCM following PI staining. (A,B) is FCM results of Apoptosis. The abscissa represents Annexin $\mathrm{V}$, and the ordinate represents PI. (C,D) is FCM results of cell cycle. The abscissa represents the DNA content and the ordinate represents the number of effective cells.

(E)The statistical results of the clones formed number in each group. (F)The statistical result of the 
numbers of cells penetrating the chamber in each group. (G)The statistical results of CCK8 assay for cell survival. (H)The formed clones of GL261 cells after crystal violet staining. (I)The microscopic image of GL261 cells penetrating the chamber after crystal violet staining $(\times 100)$. $(\mathrm{J})$ The microscopic image of GL261 cell scratch experiments. (K)The relative expression levels of Bmal1 and miR-7239-3p RNA in the two groups were detected by real-time QPCR. The internal reference protein is $\beta$-actin. $(L)$ The statistical results of cell scratch experiments. The vertical axis represents the wound healing percentage. (M)Protein bands of relevant proteins in the two groups were detected by Western blot. (N)Quantitative analysis of protein expressions. ${ }^{*} \mathrm{p} \otimes 0.05,{ }^{*} \mathrm{p} \otimes 0.01,{ }^{* \star *} \mathrm{p} \otimes 0.001$.
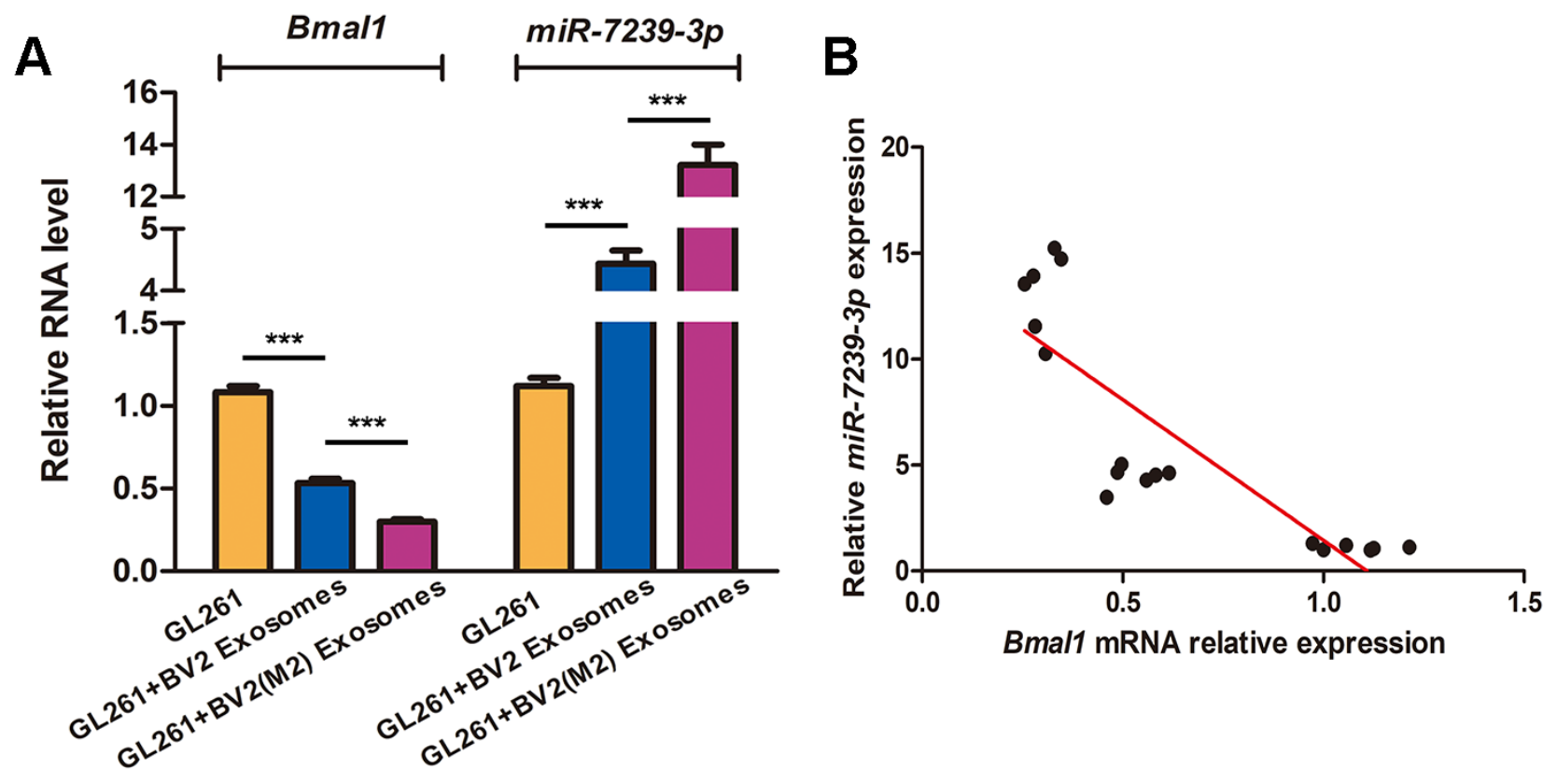

\section{Figure 7}

Real-time QPCR was used to verify relative expressions of Bmal1 and miR-7239-3p in tumor tissues. The internal reference gene were $\beta$-actin and U6 respectively. (A) shows the relative expression level of RNA. (B) is the fitting curve of the correlation analysis between miR-7239-3p and Bmal1. ${ }^{\star \star \star} p<0.001$. 


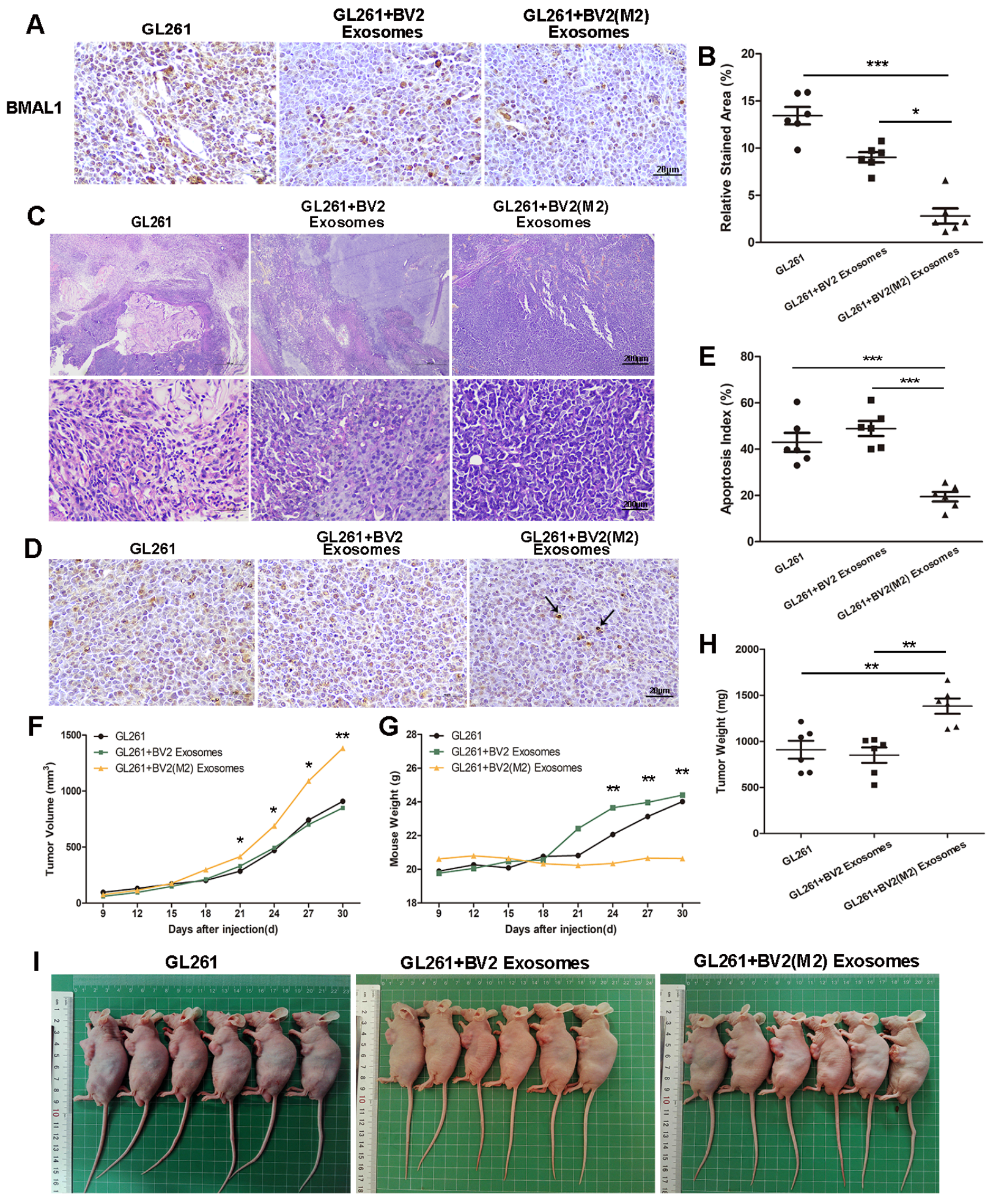

Figure 8

M2 phenotype microglia exosomes promote glioma growth in vivo. $(A, B) I H C$ was used to detect the expression of BMAL1 protein in each group. (C) The pathological morphology of glioma tissue after HE staining. (D) The TUNEL apoptosis staining of each group. (E) The statistical analysis results of apoptosis index. The arrows indicate apoptosis bodies. (F) The curve graph of tumor volume changes with time in each group. $(\mathrm{G})$ The curve of mouse weight change with time in each group. $(\mathrm{H})$ The tumor 
wights in each group. (I) The subcutaneous tumor formation in nude mice. ${ }^{*} p<0.05,{ }^{*} p<0.01$, $\star \star \star x<0.001$.

\section{Supplementary Files}

This is a list of supplementary files associated with this preprint. Click to download.

- SupplementaryTable1.doc 